\title{
Effectiveness of Shield Termination Techniques Tested with TEM Cell and Bulk Current Injection
}

\author{
Arthur T. Bradley ${ }^{\# 1}$, Richard J. Hare ${ }^{\# 2}$ \\ NASA Langley Research Center \\ 5 North Dryden, MS488, Hampton, VA 23681 USA \\ ${ }^{1}$ arthur.t.bradley@nasa.gov \\ ${ }^{2}$ richard.j.hare@nasa.gov
}

\begin{abstract}
This paper presents experimental results of the effectiveness of various shield termination techniques. Each termination technique is evaluated by two independent noise injection methods; transverse electromagnetic (TEM) cell operated from $3 \mathrm{MHz}-400 \mathrm{MHz}$, and bulk current injection (BCI) operated from $50 \mathrm{kHz}-400 \mathrm{MHz}$. Both single carrier and broadband injection tests were investigated. Recommendations as to how to achieve the best shield transfer impedance (i.e. reduced coupled noise) are made based on the empirical data. Finally, the noise injection techniques themselves are indirectly evaluated by comparing the results obtained from the TEM Cell to those from BCI .
\end{abstract}

\section{INTRODUCTION}

Cable shield termination techniques are investigated across a relative broad frequency range $(50 \mathrm{kHz}-400 \mathrm{MHz})$. The effectiveness of various termination methods were examined including pigtails, continuous shield, overbraid, and conductive tape. In addition to termination of single shields, we also investigated the effectiveness of double shields and their associated connections. Both single carrier and broadband injection tests were done.

Testing was accomplished using two injection methods; a transverse electromagnetic (TEM) cell, and bulk current injection (BCI) probes. For the case of TEM-cell testing, shielded cables were exposed to true TEM waves at frequencies ranging from $3 \mathrm{MHz}-400 \mathrm{MHz}$. The lower frequency limit was set by minimum detectable signal for the given cable length. The upper limit was set by TEM cell limitations. For the case of BCI, cables were exposed to inductive coupling at frequencies ranging from $50 \mathrm{kHz}-400$ $\mathrm{MHz}$. The performance of the probes dictated the allowed frequency range. The electronic system used for both methods of testing is kept the same and is representative of a simple real world system.

\section{EXPERIMENT SETUP}

\section{A. Electronic System}

All experiments were conducted with the same electronic system, consisting of two Hammond shielded electrical enclosures, one containing the source resistance, and the other containing the load resistance. The boxes were mounted on a large aluminium plate acting as the system chassis. Cables connecting the two boxes measured $50 \mathrm{~cm}$ in length and were attached to the boxes using D38999 military-style connectors.

For each injection method, the noise signal was created using an HP8657B signal generator or Tektronix AFG 3252 Function Generator (used for broadband noise injection experiments) and an AR 50WD1000 broadband amplifier. Measurements are taken across the load resistor using an Agilent E4401B spectrum analyzer and LeCroy AP034 active differential probe.

\section{B. TEM Cell noise injection method}

As shown in Fig. 1, the AR TC3020A TEM cell provides an environment where the entire electrical system can be exposed to radiated transverse electromagnetic fields emulating far field exposure. To achieve meaningful and repeatable data from TEM-cell experiments, two criteria were set.

1. Radiated field patterns and levels remain same throughout all tests.

2. Noise coupled into the cable (the experimental variable of interest) must dominate the inherent noise coupled through the test setup.

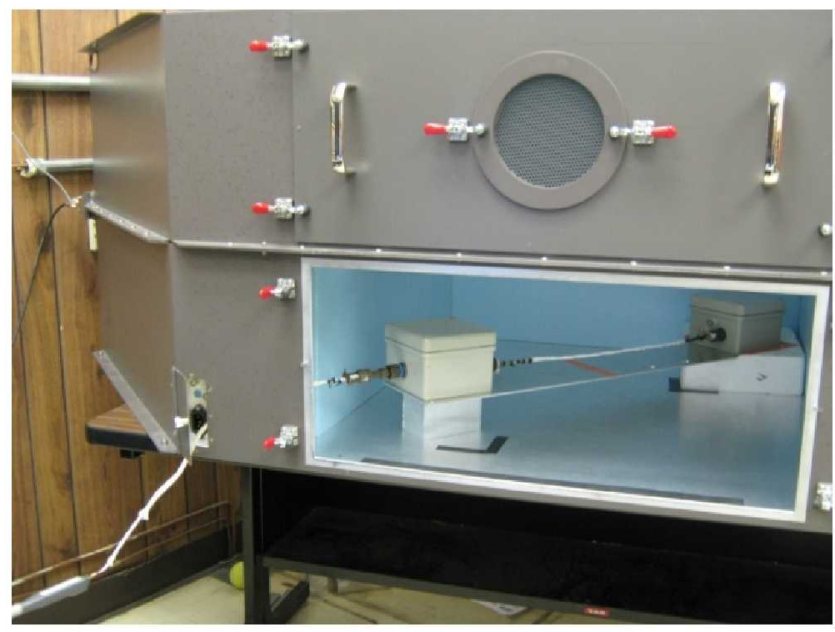

Fig. 1 TEM Cell test setup with electronic system 
The first criteria is met by fixing the position of the electronic system in the TEM cell and maintaining the same noise signal level for all tests. The second criteria is achieved by ensuring the electronic system is grounded properly, and the cable relaying the measured signal is short in length and carefully shielded. For our experiments, the electronic system is grounded on the load end through an aluminium block to the TEM cell ground plane while floating the source end of the system with a Styrofoam spacer. This ensures that there is no ground loop between the TEM cell ground plane and the aluminium chassis of the electronic system. To further minimize noise coupling into the measurement cable, an overbraid is applied to the cable and terminated into both the EMI $360^{\circ}$ backshell of the connector (grounded to the load box of the electronic system) and the TEM cell ground plane (as the cable exits the TEM Cell enclosure).

\section{BCI noise injection method}

As shown in Fig. 2, BCI probes ETS95236-1/95242-1 were used to inductively couple signals over the frequency range of interest $(50 \mathrm{kHz}-400 \mathrm{MHz})$. Due to the local nature of field exposure with the BCI method (versus the broad field exposure for the TEM Cell experimental setup), the design requirements as specified for the TEM cell can also be met for the BCI method with little trouble. The first criteria is met by keeping excitation signal levels constant through comparable tests, criteria two is met by placing the BCI clamp in the middle of the test cable.

\section{SHIELD TERMINATION METHODS}

Cable shielding often plays a crucial role in protecting electronic systems against unintentional radiation and reception of EM interference. It is well understood (although the methods are not always agreed upon) that proper shield termination significantly affects the shielding effectiveness [1], $[3],[8]-[10]$. Not obvious is to what degree parameters such as termination geometry and material have on shielding performance. It is not the goal of this particular study to investigate single-ended versus two-sided shield connections as this has already been reported [1]. Rather, this research focuses on shield geometries and materials from a practical standpoint.

\section{A. Termination Geometries and Materials}

Two predominant termination geometries are $360^{\circ}$ and single-point. Single point terminations, such as pigtails or drain wires, suffer from several shortcomings. First, they force shield current to flow in an asymmetrical manner yielding higher transfer impedances. The unshielded wiring (and associated loop) also acts as a receptor and/or radiator of noise. One would therefore predict that the pigtail termination (the only single point termination method tested in this study) would underperform the $360^{\circ}$ terminations.

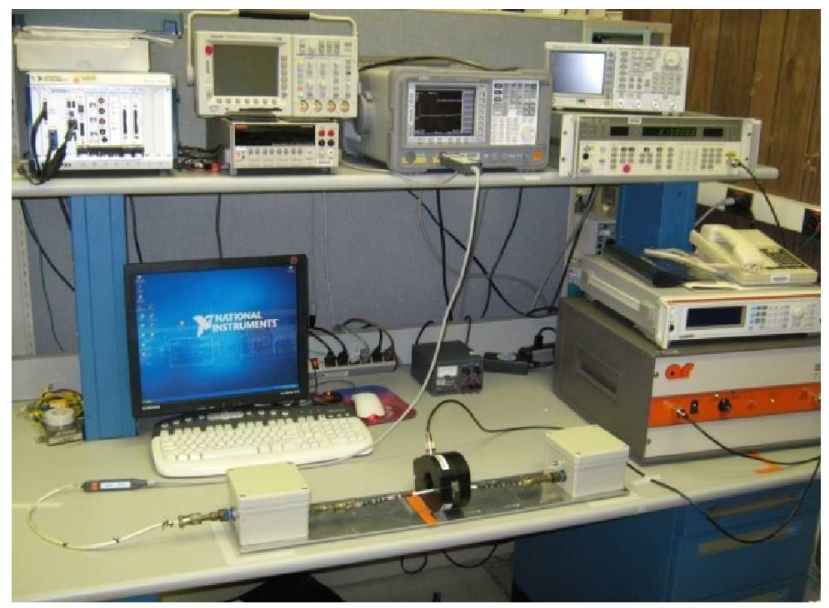

Fig. 2 BCI test setup with electronic system

Full $360^{\circ}$ shield termination may be defined as any method that terminates the shield in a circumferential manner - that is no length of the cable left exposed even as it enters the connector. Examples of $360^{\circ}$ termination geometries that were tested, include:

- cable shield expanded over an EMI backshell,

- copper tape connecting the cable shield to a standard backshell,

- overbraid secured to the cable shield and a standard connector,

- overbaid secured to the cable shield and an EMI backshell.

One would expect that any high conductivity, non-ferrous material of adequate thickness (greater than skin depth) would offer comparable shielding. Our investigation used three materials: the cable shield itself(MIL-W-1687817), shielding overbraid (RG 174), and conductive foil tape (3M 1245). In all cases, care was taken when building the cables to ensure solid mechanical and electrical contact between all conductive surfaces. Fig. 4 shows the terminations tested, where both ends of the cable are terminated in the same manner.

Fig. 3 illustrates the schematic for our electronic system. Both ends of the shield are terminated to the chassis of the electrical system through $Z_{1}, Z_{2}$. As seen from later results, the inherent impedance of each type of termination affects the shielding effectiveness of the cable. Each circuit is also grounded to chassis with the noise voltage being measured across the load resistor.

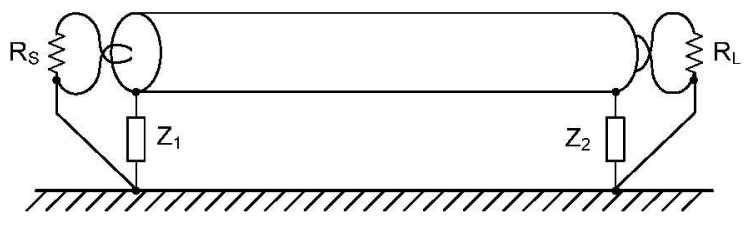

Fig. 3 Shield Termination Impedances for Twisted Shielded Pair 

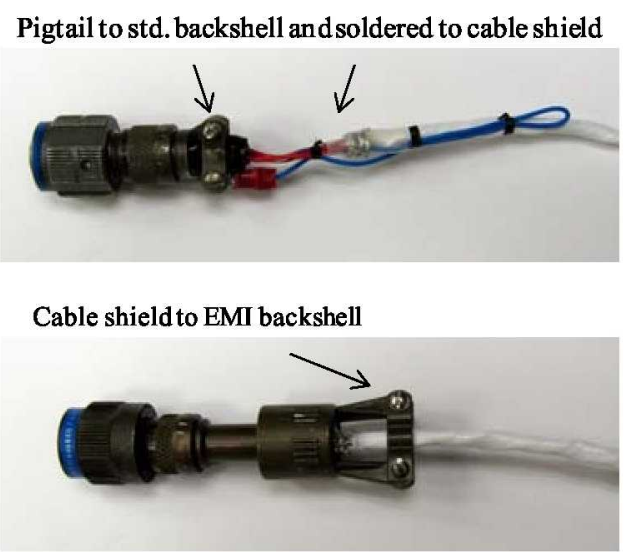

Tape wrapped over std. backshell and cable shield

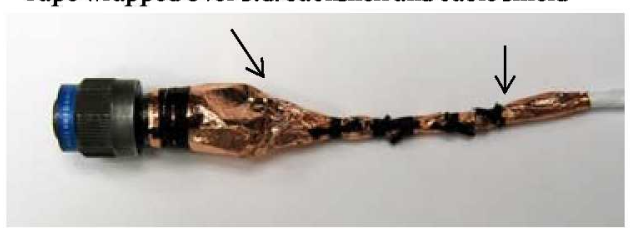

Overbraid clamped to connector - laced over cable shield

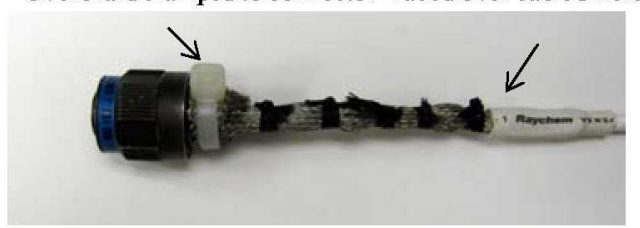

Overbraid to EMI backshell - laced over cable shield

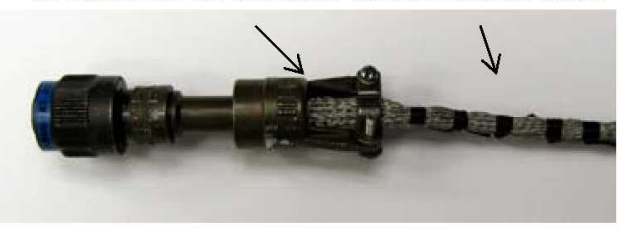

Fig. 4 Shield terminations

\section{B. TEM Cell Experimental Data-Single Shield}

It has been shown in a previous study that pigtail shield terminations are generally less effective than EMI $360^{\circ}$ backshell terminations [1]. One would therefore expect that all of the $360^{\circ}$ termination methods would show improvement in shield performance over the pigtail case. Of greater interest however is how the different methods of achieving the $360^{\circ}$ affect the shielding effectiveness.

Fig. 5 and Fig. 6 show the results from TEM-cell testing of the five different termination types. Across the majority of the test frequency range, the pigtail termination is approximately $30 \mathrm{~dB}$ worse than all other types. It is interesting to note that there is little difference in shielding performance between any of the $360^{\circ}$ termination methods. This is significant, because it suggests that the use of non-optimal $360^{\circ}$ terminations (e.g. conductive tape) as can be quite effective.

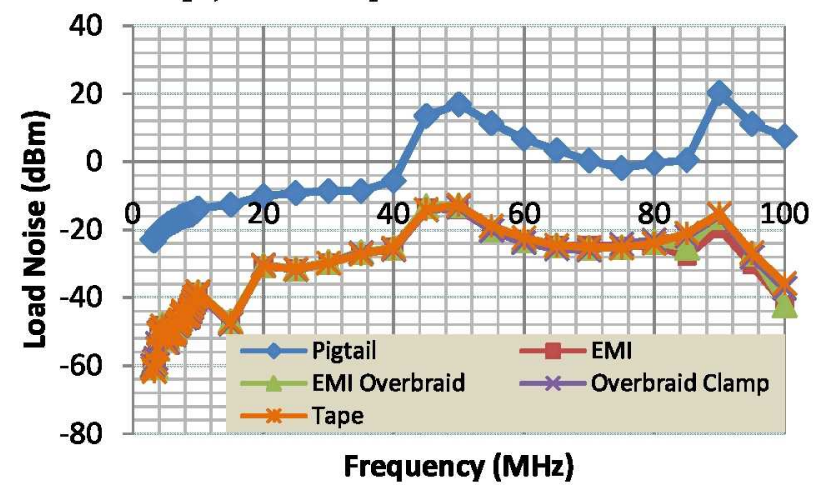

Fig. 5 TEM-cell-shield termination effectiveness $(3-100 \mathrm{MHz})$

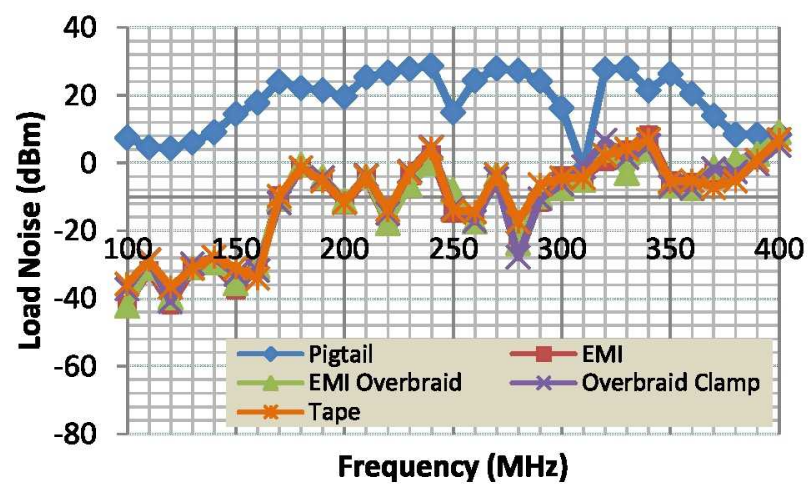

Fig. 6 TEM-cell tested shield termination effectiveness $(100-400 \mathrm{MHz})$

\section{BCI Experimental Data-Single Shield}

As shown in Fig. 7, the results from BCI testing are similar to that from the TEM cell for the comparable rage of $3 \mathrm{MHz}$ to $400 \mathrm{MHz}$. BCI testing demonstrates that all the $360^{\circ}$ terminations exceed that of the pigtail by approximately $40 \mathrm{~dB}$.

In utilizing inductive coupling, BCI testing allows the effects of much lower noise frequencies to be measured. In our particular study, testing at lower frequencies better emphasizes the resistive component of the shield termination impedance (except for the tape terminated cable, as later explained). The shielding effectiveness at lower to midrange frequencies $(0.05-25 \mathrm{MHz})$ is given in Fig. 8 .

The best performing cables (from $50 \mathrm{kHz}$ to $5 \mathrm{MHz}$ ) listed in order of shielding effectiveness are: EMI overbraid, EMI, overbraid tied to connector, conductive tape, and pigtail. It is interesting to note that the conductive tape-terminated cable 


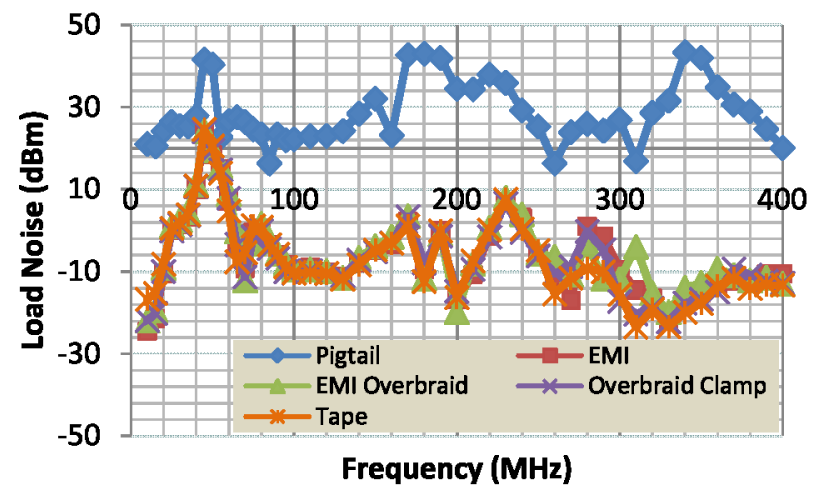

Fig. 7 BCI-tested shield termination effectiveness $(0.05-400 \mathrm{MHz})$

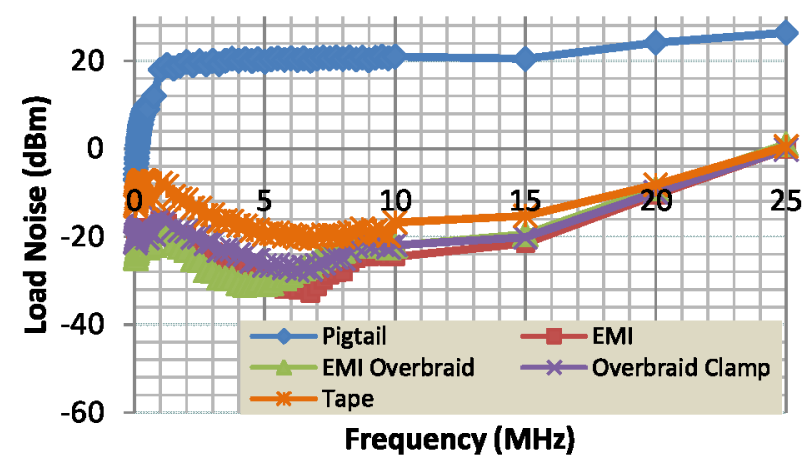

Fig. 8 BCI-tested shield termination effectiveness $(0.05-25 \mathrm{MHz})$

performs approximately $10 \mathrm{~dB}$ worse than the other tested $360^{\circ}$ terminations for this frequency range. After $5 \mathrm{MHz}$ this difference decreases, until the tape-terminated cable is approximately equal in effectiveness at about $20 \mathrm{MHz}$. This phenomenon can be explained when the actual geometry and properties of the tape termination are considered.

Geometrically, the tape is spirally wound with overlapping turns around the cable shield and connector backshell. While these turns make electrical contact, there still exists some capacitance between each layer of overlapped foil due to the adhesive. This finite capacitance forces lower frequency shield current to flow spirally through the tape termination creating a mutual inductance between the tape termination and the shielded conductors within. At around $20 \mathrm{MHz}$, the capacitive reactance is small enough to effectively short the turns of the tape. This causes the copper tape to electrically look more like a $360^{\circ}$ conductive cylindrical shield [3].

\section{Broadband Data-Single Shield}

Unlike the swept single-carrier data presented in sections $A$.-C., the following data is obtained through driving the TEM cell and BCI probes with a broadband signal (periodic pulse of $5 \mathrm{MHz}$ repetition rate, $2.5 \mathrm{~ns}$ rise/fall times, and $4 \mathrm{~ns}$ pulse width). The narrow pulse width and short rise and fall times create a broadband distribution spectral energy.

The broadband results for both TEM-cell and BCI testing are given in Table I. Once again there is a clear distinction between effectiveness of the pigtail and $360^{\circ}$ terminations. Once again, all of the $360^{\circ}$ termination methods yielded nearly identical shielding properties to the net broadband energy.

TABLE I

BROADBAND NOISE INJECTION-SHIELD TERMINATION METHODS

\begin{tabular}{|c|c|c|}
\hline Cable Type & $\begin{array}{l}\text { TEM } \\
\text { (dB) }\end{array}$ & $\begin{array}{l}\text { BCI } \\
\text { (dB) }\end{array}$ \\
\hline Pigtail & -7.10 & 1.26 \\
\hline EMI & -24.58 & -17.68 \\
\hline EMI Overbraid & -25.31 & -17.68 \\
\hline Overbraid bonded & -24.47 & -17.98 \\
\hline Tape & -24.24 & -17.68 \\
\hline
\end{tabular}

\section{DOUBLE SHIELD TERMINATIONS}

In situations where additional EM protection is needed, double shielded cables can be employed. Other authors have concluded that two braided shields can help to reduce noise coupling by 20 to $30 \mathrm{~dB}$ [3]. While it is not our intention to compare single shield effectiveness against double shield effectiveness we do wish to compare the relative effectiveness of three specific double shield termination strategies as shown in Table II.

TABLE II

DOUBLE SHIELD TERMINATION CABLE TYPES

\begin{tabular}{|c|l|}
\hline SS_SS & $\begin{array}{l}\text { Description } \\
\text { EMI } 360 \text { backshell with overbraid. }\end{array}$ \\
\hline SO_OS & $\begin{array}{l}\text { One end of the cable has the outer shield } \\
\text { terminated to EMI 360 backshell with } \\
\text { overbraid, and the inner shield is left } \\
\text { unterminated. }\end{array}$ \\
\hline S.TP_S.TP & $\begin{array}{l}\text { Opposite end of cable has the outer shield } \\
\text { unterminated and the inner shield terminated } \\
\text { to EMI 360 backshell with overbraid. }\end{array}$ \\
$\begin{array}{l}\text { Outer shields are terminated to EMI 360 } \\
\text { backshell with overbraid, and the inner shields } \\
\text { are terminated to a quiet ground on the } \\
\text { source/load circuits using a through pin } \\
\text { connection }\end{array}$ \\
\hline
\end{tabular}

It is important to note that all three cables were made from the same double shielded double jacketed stock cable (Blake M27500-22-NE-2-A72) using identical connectors, EMI 360 backshells, and overbraid.

\section{A. TEM Cell Experimental Data-Double Shield}

When predicting the effectiveness of each of the three cable types, it is important to notice that the SS_SS and S.TP_S.TP cables have shields tied to chassis at both ends. At low frequencies, the SO_OS cable will prevent the flow of shield current. However at higher frequencies, it should act similar to 
the shorted cables because the capacitive coupling will provide an effective short between the shield layers.

This predicted behaviour is evident in Fig. 9 and Fig. 10 as the SO_OS cable provides about $45 \mathrm{~dB}$ less protection when compared to the SS_SS and S.TP_S.TP cables. This trend continues until approximately $150^{-} \mathrm{MHz}$ where the noise frequency is sufficient to allow for capacitive coupling in the SO OS cable. While not necessarily evident from Fig. 10, there is a $4 \mathrm{~dB}$ improvement of the S.TP_S.TP cable over the SS_SS cable until around $30 \mathrm{MHz}$. A After this crossover frequency it seems that the S.TP_S.TP cable performs slightly worse than the SS_SS. This data would suggest that for frequencies below $10-30 \mathrm{MHz}$, there is a slight advantage to routing the inner shield through a pin to an internal system ground. That advantage is reversed at higher frequencies.

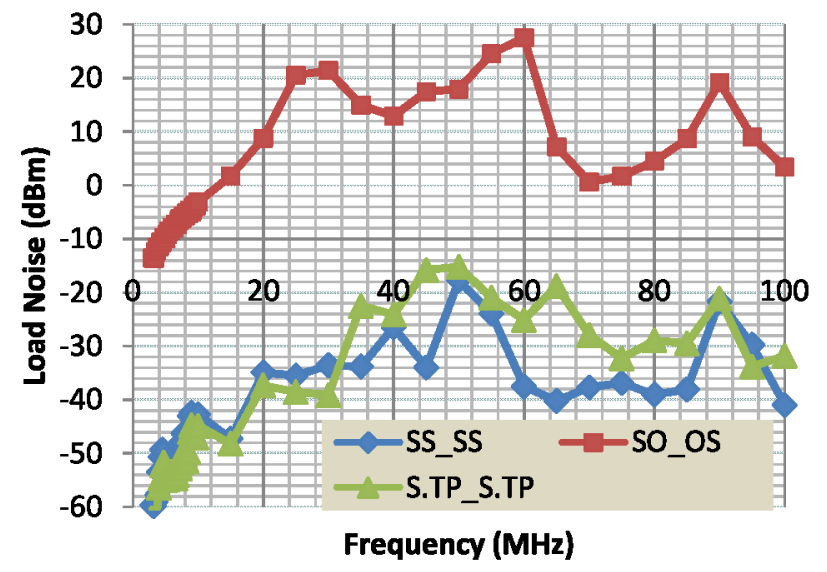

Fig. 9 TEM Cell-Double Shield Effectiveness (3- $100 \mathrm{MHz}$ )

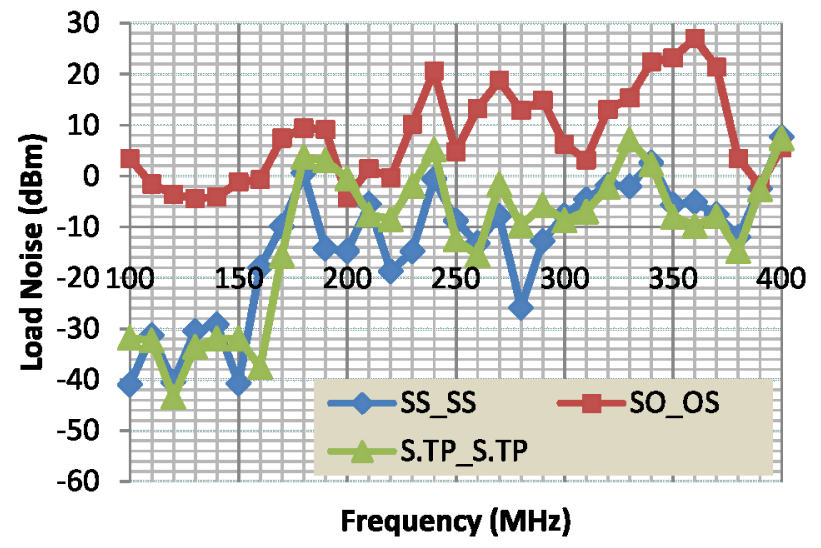

Fig. 10 TEM Cell-Double Shield Effectiveness (100- $400 \mathrm{MHz})$

\section{B. BCI Experimental Data-Double Shield}

Bulk current injection testing yields comparable results as seen in Fig. 11 and Fig. 12. In this test the SO_OS cable also performs worse at lower frequencies. Also similar to the TEM cell data is the $4 \mathrm{~dB}$ improvement of the through pin cable versus the SS_SS cable until around $30 \mathrm{MHz}$, after which no consistent benefit is realized.

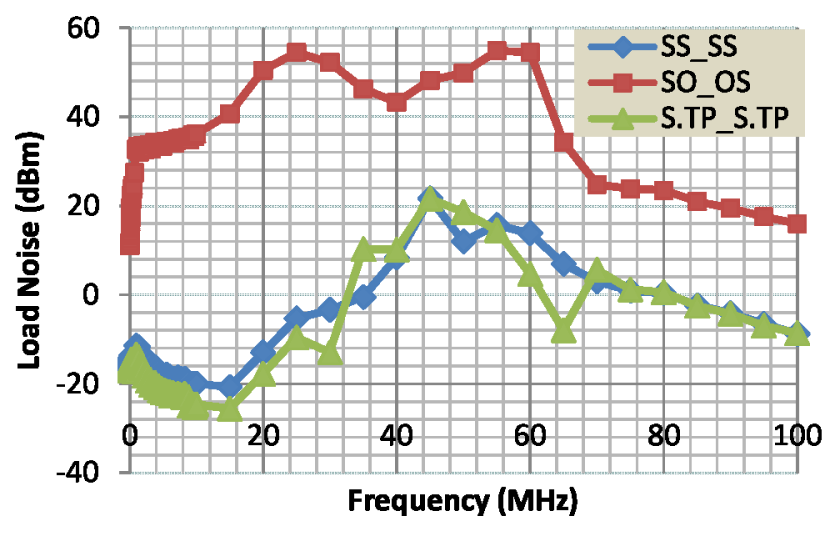

Fig. 11 BCI-Double Shield Effectiveness (0.05- $100 \mathrm{MHz})$

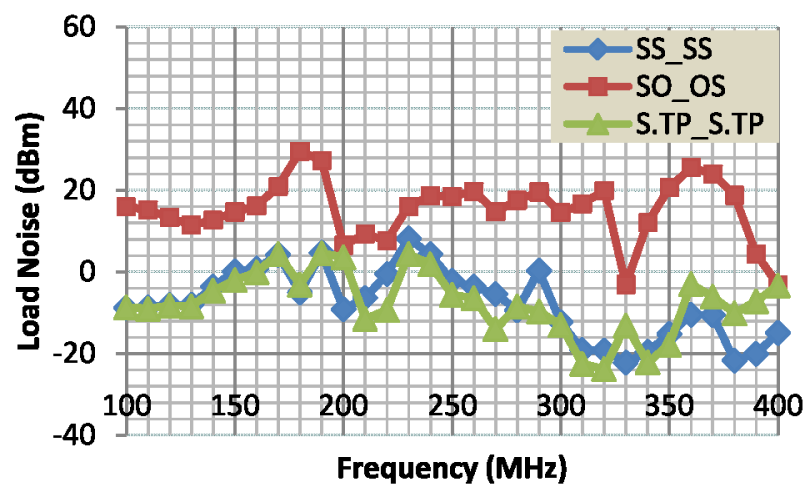

Fig. 12 BCI-Double Shield Effectiveness (100- $400 \mathrm{MHz})$

\section{CONCLUSIONS}

Many options exist for shielding termination, including pigtails, overbraid, and conventional $360^{\circ}$ EMI backshells. Although it has long been known that pigtail shield connections underperform $360^{\circ}$ termination methods, our experiments indicated that pigtails were typically worse by more than $35 \mathrm{~dB}$ for frequencies above a few $\mathrm{MHz}$. This would indicate that one should avoid terminating shields with pigtail connections. However if a pigtail termination is to be used, shortening the pigtail wire length and minimizing exposed wiring has been shown to slightly improve the noise rejection capabilities of the cable [1].

All TEM cell and BCI data suggest any of the four tested $360^{\circ}$ shield termination techniques provide comparable protection for frequencies above $20 \mathrm{MHz}$. This is primarily due to the circumferential nature of all terminations where virtually no exposed wiring exists and inductive effects are minimized. However, from $50 \mathrm{kHz}$ to $20 \mathrm{MHz}$ BCI testing indicates that a foil tape shield termination is less effective (as much as $10 \mathrm{~dB}$ ) when compared to the other $360^{\circ}$ terminations. This may be related to both the mutual inductance between the tape and inner shielded conductors and the finite capacitance between the layers of the tape.

When single shielded cable assemblies do not provide the necessary EMI protection, double shielded cables can yield 
additional noise rejection capabilities [3]. Of the three tested double-shield configurations, the S.TP_S.TP cable performed the best at frequencies below $30 \mathrm{MHz}$, above which it was slightly inferior to the SS SS cable. The SO OS cable offered significantly less protection, and in general should only be used when preventing dc shield current is a necessity (i.e. worried about low-frequency ground loops).

Comparing the results from the TEM cell and BCI methods, both methods yielded very similar results for our simple electrical system. This high degree of correlation would lead to the conclusion that both methods are valid when testing for cable susceptibility up to a few hundred $\mathrm{MHz}$. Bulk current injection has the additional benefit of being able to couple EM energy into a system at lower frequencies. However this method constitutes a local inductive injection of noise and does not always accurate reflect the benefits if shielding against electric fields. TEM cell exposure subjects an entire electrical system to true far-field radiated emissions, at the expense of size restrictions and more complicated test setup requirements.

\section{REFERENCES}

[1] A..T. Bradley, TEM Cell Testing of Cable Noise Reduction Techniques from $2 \mathrm{MHz}$ to $200 \mathrm{MHz}-$ Part 1, 2008 Asia Pacific EMC Symposium, Singapore, 2008.

[2] A..T. Bradley, TEM Cell Testing of Cable Noise Reduction Techniques from $2 \mathrm{MHz}$ to $200 \mathrm{MHz}-$ Part 2, 2008 Asia Pacific EMC Symposium, Singapore, 2008

[3] E.F. Vance, Coupling to Shielded Cables, Wiley Interscience, New York, 1978.

[4] C.R. Paul, Introduction to Electromagnetic Compatibility, $2^{\text {nd }}$ ed., Wiley Interscience, New Jersey, 2006.

[5] H.W. Ott, Noise Reduction Techniques in Electronic Systems, $2^{\text {nd }}$ ed., Wiley Interscience, New York, 1988.

[6] D.A. Weston, Electromagnetic Compatibility Principles and Applications, $2^{\text {nd }}$ ed., Marcel Dekker, New York, 2001.

[7] D.H. Trout, N.F. Audeh, Evaluation of Electromagnetic Radiated Susceptibility Testing using Induced Currents, Aerospace Conference, 1997.

[8] M. Mardiguian, Handbook Series on Electromagnetic Interference and Compatibility: Volume 2 - Grounding and Bonding, Interference Control Technologies, Virginia, 1988.

[9] R.B. Schulz, V.C. Plantz, and D.R. Brush, Shielding Theory and Practice, IEEE Transactions on Electromagnetic Compatibility, Vol. 30 No. 3, August 1988

[10] K.L. Kaiser, Electromagnetic Compatibility Handbook, CRC Press, 2005.

[11] H.W. Ott, Ground-a Path for Current Flow, IEEE Proceedings Int. Symposium Electromagnetic Compatibility, San Diego, CA 1979. 